Wilfrid Laurier University

Scholars Commons @ Laurier

Physics and Computer Science Faculty

Publications

Physics and Computer Science

$4-2008$

\title{
Simultaneous Measurement for Strain and Temperature Using Fiber Bragg Gratings and Multimode Fibers
}

\author{
Da-Peng Zhou \\ University of Waterloo \\ Li Wei \\ Wilfrid Laurier University, Iwei@wlu.ca \\ Wing-Ki Liu \\ University of Waterloo \\ John W.Y. Lit \\ Wilfrid Laurier University, jlit@wlu.ca
}

Follow this and additional works at: https://scholars.wlu.ca/phys_faculty

\section{Recommended Citation}

Zhou, Da-Peng; Wei, Li; Liu, Wing-Ki; and Lit, John W.Y., "Simultaneous Measurement for Strain and Temperature Using Fiber Bragg Gratings and Multimode Fibers" (2008). Physics and Computer Science Faculty Publications. 17.

https://scholars.wlu.ca/phys_faculty/17

This Article is brought to you for free and open access by the Physics and Computer Science at Scholars Commons @ Laurier. It has been accepted for inclusion in Physics and Computer Science Faculty Publications by an authorized administrator of Scholars Commons @ Laurier. For more information, please contact scholarscommons@wlu.ca. 


\title{
Simultaneous measurement for strain and temperature using fiber Bragg gratings and multimode fibers
}

\author{
Da-Peng Zhou, ${ }^{1, \star}$ Li Wei, ${ }^{2}$ Wing-Ki Liu, ${ }^{1}$ Yu Liu, ${ }^{2}$ and John W. Y. Lit ${ }^{2}$ \\ 'Department of Physics and Astronomy, Guelph-Waterloo Physics Institute,University of Waterloo, \\ Waterloo, Ontario N2L 3G1, Canada \\ ${ }^{2}$ Department of Physics and Computer Science, Wilfrid Laurier University, Waterloo, Ontario N2L 3C5, Canada \\ *Corresponding author: d4zhou@uwaterloo.ca
}

Received 26 November 2007; revised 22 January 2008; accepted 16 February 2008; posted 21 February 2008 (Doc. ID 90078); published 31 March 2008

\begin{abstract}
An all-fiber sensor capable of simultaneous measurement of temperature and strain is newly presented. The sensing head is formed by a fiber Bragg grating combined with a section of multimode fiber that acts as a Mach-Zehnder interferometer for temperature and strain discrimination. The strain and temperature coefficients of multimode fibers vary with the core sizes and materials. This feature can be used to improve the strain and temperature resolution by suitably choosing the multimode fiber. For a $10 \mathrm{pm}$ wavelength resolution, a resolution of $9.21 \mu \varepsilon$ in strain and $0.26{ }^{\circ} \mathrm{C}$ in temperature can be achieved.

(C) 2008 Optical Society of America

OCIS codes: $\quad 060.2310,060.2370$.
\end{abstract}

\section{Introduction}

There has been considerable interest in developing methods that enable optical fibers to measure strain and temperature simultaneously [1-13]. These fiberoptic sensors are usually based on detecting two physical parameters that have different sensitivities to temperature and strain. Many techniques based on fiber Bragg gratings (FBGs) have been reported for simultaneous strain and temperature discrimination, such as using two superimposed FBGs $[\underline{3}, \underline{4}]$, two resolvable wavelengths in a tilted FBG [5], two FBGs written in fibers of different diameters [6] or compositions [7], and a single FBG written across a splice point between fibers of different refractive indices [8] or between fibers with different levels of doping elements [9]. Alternatively, the measurement from an FBG may be combined with that of a different sensing technique, such as using hybrid FBG/longperiod grating [10], superstructure FBG [11], a sampled FBG combined with a long-period grating

0003-6935/08/101668-05\$15.00/0

(C) 2008 Optical Society of America
[12], and an FBG combined with a polarizationmaintaining fiber loop mirror [13].

On the other hand, all-fiber Mach-Zehnder interferometer (MZI) sensors based on mode interference have increasingly aroused research interest in the past several years, because they are compact and easy to fabricate. For example, Wang and Farrell proposed an all-fiber multimode-interference-based refractometer for chemical sensors [14], Mehta et al. investigated multimode-interference-based MZIs for displacement sensors [15], and Frazão et al. demonstrated a curvature sensor based on multimode interference combined with a long-period grating [16]. Temperature and strain sensors based on all-fiber MZIs were also widely studied, but employing different multimode fibers (MMFs) [17-20]. Since MMF has strain and temperature sensitivity responses different from those of the FBG, it is feasible to realize simultaneous measurement of the strain and temperature variations based on their combination.

In this paper, we present an all-fiber sensor for simultaneous strain and temperature discrimination using an FBG combined with a section of MMF as a sensing head. The MMF acts as an MZI and the interference fringes are sensitive to the applied 
strain and ambient temperature. By measuring the resonance wavelength shifts of the MMF and the FBG, the applied strain and temperature changes can be determined simultaneously. The sensor resolution depends on not only the sensitivities of the individual sensor heads but also their combination. Three kinds of MMFs combined with one FBG were used in our experiment, and the sensitivity performances of the three combinations were investigated. The experimental results show that the strain and temperature coefficients of the MMF are very different for different fiber core sizes and materials, and high resolution could be achieved by selecting a suitable combination of MMF and FBG. This feature may provide a new approach to optimize the sensor resolution.

\section{Operation Principle and Experiment Setup}

As shown in Fig. 1, the FBG and MMF are spliced to form an all-fiber transmission-type sensor. The FBG was written in SMF-28. The section of MMF acts as an MZI. The fundamental mode that propagates along the single-mode fiber (SMF) will couple into the MMF at splice point $\mathrm{A}$, and the power will be dominantly distributed in the lowest-order mode, while only a small portion of power propagates in the higher-order modes. The different modes will interfere and recouple back into the SMF at splice point $\mathrm{B}$. When only the first two lowest-order modes are considered, the measured intensity is given by

$$
I=I_{1}+I_{2}+2 \sqrt{I_{1} I_{2}} \cos \left(\frac{2 \pi \Delta n L}{\lambda}\right),
$$

where $I_{1}$ and $I_{2}$ are the power distributed in the firstand the second-order modes, respectively, $I$ is the measured power, $L$ is the length of the MMF, $\lambda$ is the wavelength of light in vacuum, $\Delta n=n_{2}-n_{1}$, and $n_{1}$ and $n_{2}$ are the mode indices. The wavelength spacing $\Delta \lambda$ of the interfering spectrum is given by Eq. (1) as $\Delta \lambda \approx \lambda^{2} /(\Delta n L)$.

When a strain is applied to the MMF and FBG or an ambient temperature changes, the mode indices, the fiber length, and the grating pitch will change; therefore, the resonance wavelengths will shift. The shifts of the resonance wavelengths for the FBG and MMF can be expressed as

$$
\left[\begin{array}{l}
\Delta \lambda_{\mathrm{FBG}} \\
\Delta \lambda_{\mathrm{MMF}}
\end{array}\right]=\left[\begin{array}{ll}
\alpha_{\mathrm{FBG}} & \zeta_{\mathrm{FBG}} \\
\alpha_{\mathrm{MMF}} & \zeta_{\mathrm{MMF}}
\end{array}\right]\left[\begin{array}{l}
\epsilon \\
\Delta T
\end{array}\right]
$$

where $\Delta \lambda_{\mathrm{FBG}}$ and $\Delta \lambda_{\mathrm{MMF}}$ are FBG and MMF wavelength shifts; $\varepsilon$ and $\Delta T$ are applied strain and ambient temperature change, respectively; and $\alpha_{\mathrm{FBG}}$, $\zeta_{\mathrm{FBG}}, \alpha_{\mathrm{MMF}}$, and $\zeta_{\mathrm{MMF}}$ are the corresponding strain and temperature coefficients that can be determined by experiment. The applied strain and the temperature changes can then be simultaneously obtained from Eq. (2):

$$
\left[\begin{array}{l}
\varepsilon \\
\Delta T
\end{array}\right]=M^{-1}\left[\begin{array}{l}
\Delta \lambda_{\mathrm{FBG}} \\
\Delta \lambda_{\mathrm{MMF}}
\end{array}\right],
$$

where

$$
M^{-1}=\frac{1}{|M|}\left[\begin{array}{ll}
\zeta_{\mathrm{MMF}} & -\zeta_{\mathrm{FBG}} \\
-\alpha_{\mathrm{MMF}} & \alpha_{\mathrm{FBG}}
\end{array}\right]
$$

with $|M|=\alpha_{\mathrm{FBG}} \zeta_{\mathrm{MMF}}-\alpha_{\mathrm{MMF}} \zeta_{\mathrm{FBG}}$, the determinant of the coefficient matrix. For given wavelength measurement errors, $\delta\left(\Delta \lambda_{\mathrm{FBG}}\right)$ and $\delta\left(\Delta \lambda_{\mathrm{MMF}}\right)$, the tolerances of the measured stain and temperature are given by

$$
\left[\begin{array}{l}
\delta(\varepsilon) \\
\delta(\Delta T)
\end{array}\right]=\operatorname{abs}\left(M^{-1}\right)\left[\begin{array}{l}
\delta\left(\Delta \lambda_{\mathrm{FBG}}\right) \\
\delta\left(\Delta \lambda_{\mathrm{MMF}}\right)
\end{array}\right] .
$$

The wavelength measurement errors are determined by the resolution of the optical spectrum analyzer (OSA) and the bandwidth of the FBG and MMF resonance, which is true for all optical sensing applications. A large absolute value of the coefficient matrix determinant is usually desirable for highresolution measurement. Fortunately, we found that the strain and temperature coefficients of the MMFs could vary over a big range with the core size and material. This may provide us a very easy and costeffective approach to improve the sensing resolutions for simultaneous strain and temperature measurements by choosing the MMF with proper strain and temperature coefficients.

\section{Experiment and Discussion}

As shown in Fig. 1, the experiment setup consists of an optical broadband source, an FBG, a section of MMF and SMF, and an OSA with a resolution of $0.06 \mathrm{~nm}$. The sensing head was placed in a copper tube with a diameter of $1.5 \mathrm{~cm}$, which was wrapped by a heating tape. One side of the fiber was attached to a translation stage with a resolution of $10 \mu \mathrm{m}$. Three different kinds of MMFs, $\mathrm{MMF}_{i}(i=1,2,3)$

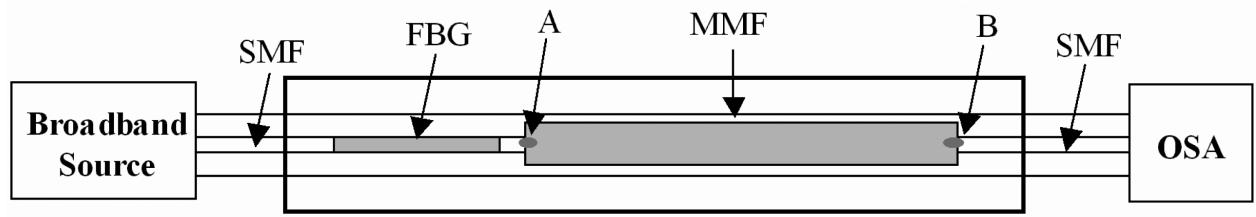

Heating tube

Fig. 1. Configuration of experimental setup consisting of an FBG, a section of MMF, SMF, a broadband light source, and an OSA. 
and an FBG with a center wavelength of $1560.05 \mathrm{~nm}$, reflectivity of $84.5 \%$, and optical bandwidth of $0.21 \mathrm{~nm}$ were used in our experiment. All three of these MMFs are commercially available. Table 1 lists the standard parameters and lengths of the $\bar{M} M F s$ used in our experiment as well as the wavelength spacing of the resultant interference spectrum. Note that $\mathrm{MMF}_{3}$ was originally designed for dispersion compensation applications, with a large dispersion parameter of $-270 \mathrm{ps} / \mathrm{nm} / \mathrm{km}$ at $1550 \mathrm{~nm}$ and a cutoff wavelength of $1663 \mathrm{~nm}$.

The transmission spectrum is shown in Fig. 2. The FBG spectrum is superimposed on the $\mathrm{MMF}_{3}$ spectrum. The extinction ratio of the interference fringes is determined by the ratio of the intensities of the two interfering modes, which can be controlled by the splicing condition. We have optimized the extinction ratio from 2 to $8 \mathrm{~dB}$ by slightly offsetting the center of the core of the SMF with respect to that of the $\mathrm{MMF}_{3}$ at point $\mathrm{A}$, but without offsetting at point $\mathrm{B}$. The extinction ratio of $8 \mathrm{~dB}$ corresponds to a relative power distribution $I_{2} /\left(I_{1}+I_{2}\right)$ of $15.2 \%$ [20]. Further improvement of the extinction ratio may be obtained by offsetting both ends, but with the compromise of the insertion loss. The applied strain and ambient temperature changes can be determined by measuring the resonance wavelength shifts of the FBG and $\mathrm{MMF}_{3}$. To determine the strain and temperature coefficients, strain and temperature changes were applied to the fiber separately. We first measure the strain responses of the $\mathrm{FBG}$ and $\mathrm{MMF}_{i}$ $(i=1,2,3)$ by measuring the wavelength shifts as a function of the applied stain under the room temperature of $22^{\circ} \mathrm{C}$, as shown in Fig. 3 . Figure 4 shows the wavelength shifts of the FBG and $\mathrm{MMF}_{i}$ with respect to temperature changes when the tube is heated from $22^{\circ} \mathrm{C}$ to $90^{\circ} \mathrm{C}$ under zero strain. The measured wavelength shifts have a good linear behavior with variations in strain and temperature. It can be seen that the strain and temperature coefficients of the MMFs are very different: there are all blueshifts for the strain responses (i.e, negative slopes), while there are one redshift and two blueshifts for the temperature responses.

For given measurement errors of wavelength shifts, the strain and temperature errors are given in Eq. (4). Assuming a wavelength resolution of $10 \mathrm{pm}$ for the FBG and MMF, the measurement errors of the strain and temperature for the three combinations are listed in Table 2 . The condition number of the coefficient matrix for each combination is also listed in Table 2 , which provides a common method of assessing the performance of a temperature-strain

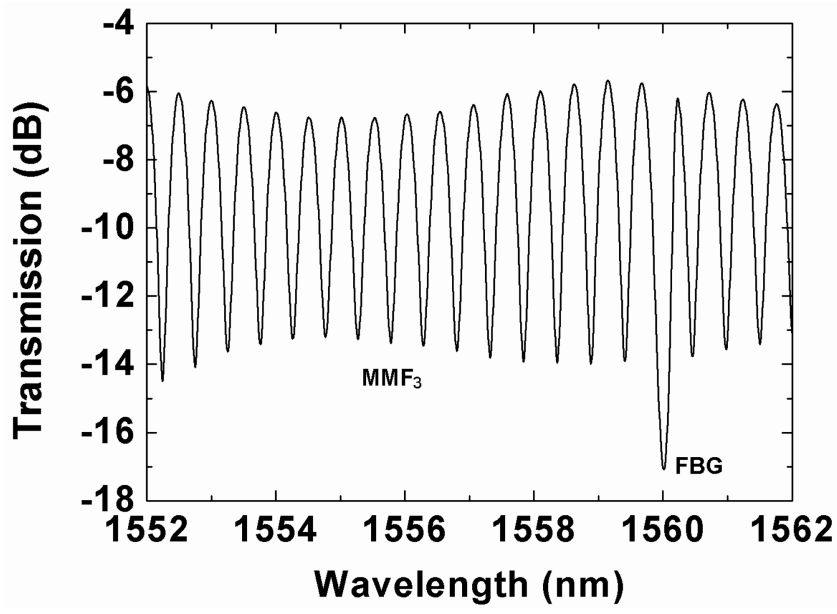

Fig. 2. Transmission spectrum shows that the FBG spectrum is superimposed on the $\mathrm{MMF}_{3}$ 's.

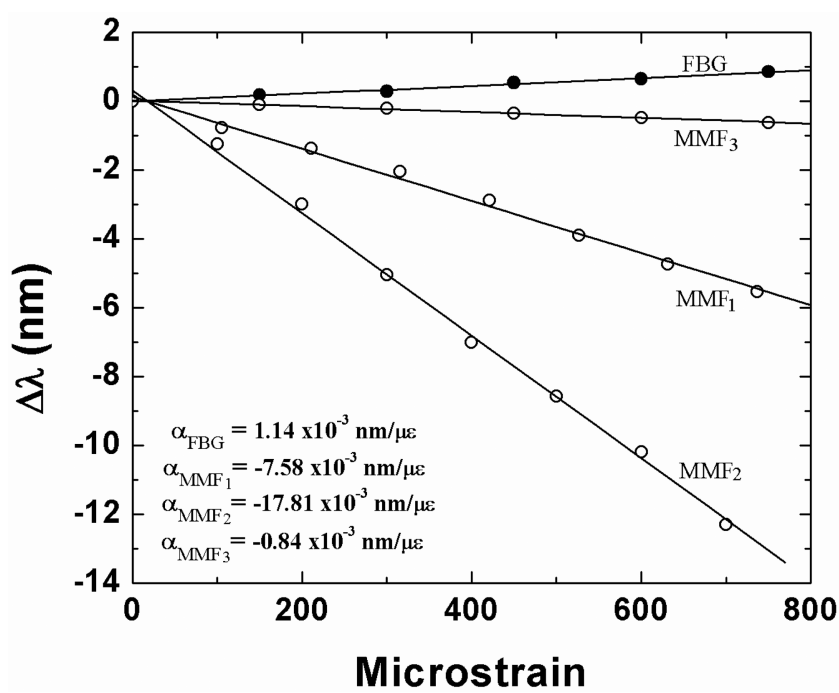

Fig. 3. Wavelength shifts as a function of applied strain for an FBG combined with three different MMFs, showing very different strain coefficients for different types of MMF.

discrimination system [21]. A smaller condition number is preferred. It is very clear that the combination of $\mathrm{FBG}+\mathrm{MMF}_{3}$ (with the smallest condition number) results in good strain and temperature resolutions of $9.21 \mu \varepsilon$ in strain and $0.26^{\circ} \mathrm{C}$ in temperature, whereas $3-10$ times resolution degradation were obtained for the other two combinations (with much greater condition numbers). Note that for

Table 1. Typical Parameters and Segment Lengths of MMFs Used in the Experiment and Wavelength Spacing of the Resultant Interference Spectrum

\begin{tabular}{cccccc}
\hline Fiber Type & Numerical Aperture & Core/Cladding Diameter $(\mu \mathrm{m})$ & Length $(\mathrm{m})$ & Wavelength Spacing $(\mathrm{nm})$ & Company \\
\hline $\mathrm{MMF}_{1}$ & 0.29 & $100 / 140$ & 1.4 & 6.4 & POFC \\
$\mathrm{MMF}_{2}$ & 0.275 & $62.5 / 125$ & 1.4 & 6.8 & POFC \\
$\mathrm{MMF}_{3}$ & 0.342 & $1.9 / 115.7$ & 0.24 & 0.5 & Sumitomo Electric \\
\hline
\end{tabular}




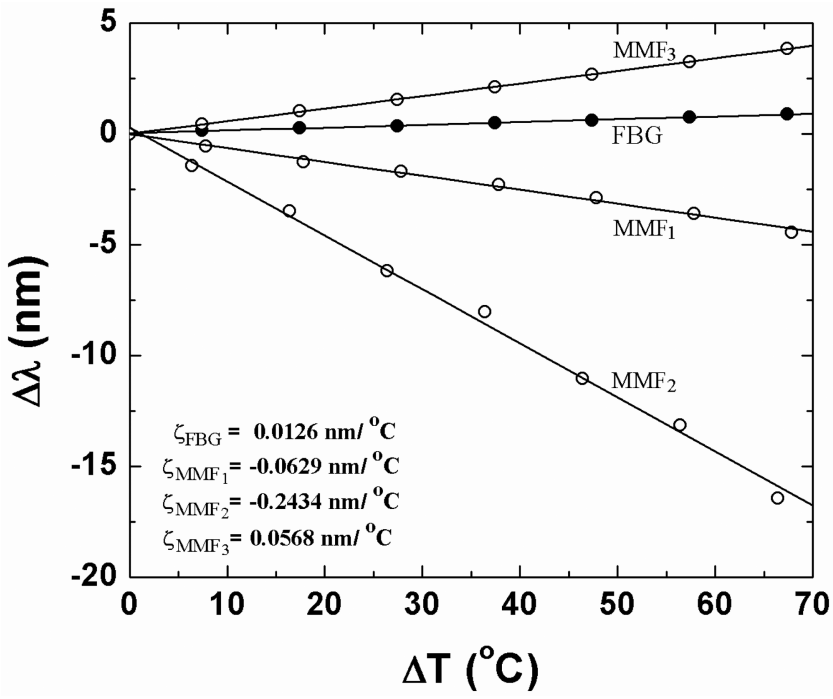

Fig. 4. Wavelength shifts as a function of ambient temperature for an FBG combined with three types of MMFs, showing very different temperature coefficients for different types of MMF.

the combination of $\mathrm{FBG}+\mathrm{MMF}_{3}$, the strain responses are in opposite directions (i.e., one has a blueshift and the other has a redshift), and the temperature responses are in the same direction (i.e., both have a redshift). This suggests that when using the combination of two types of fiber-based elements with similar sensitivities as a sensing head for simultaneous measurement of temperature and strain, it is desirable to choose the two elements with opposing trends in one type of measurement (e.g., strain) and similar but distinct trends in the other type of measurement (e.g., temperature). For the proposed structure based on MMF and FBG, by properly selecting the MMF, one may obtain a high sensing resolution for simultaneous strain and temperature measurement.

For the combination of $\mathrm{FBG}+\mathrm{MMF}_{3}$, the coefficient matrix is listed in Table 2. By using Eq. (3), the simultaneous measurement of strain and temperature is shown in Fig. $\underline{5}$. There are two planes, individually indicating the applied strain and temperature changes. For a given pair of wavelength

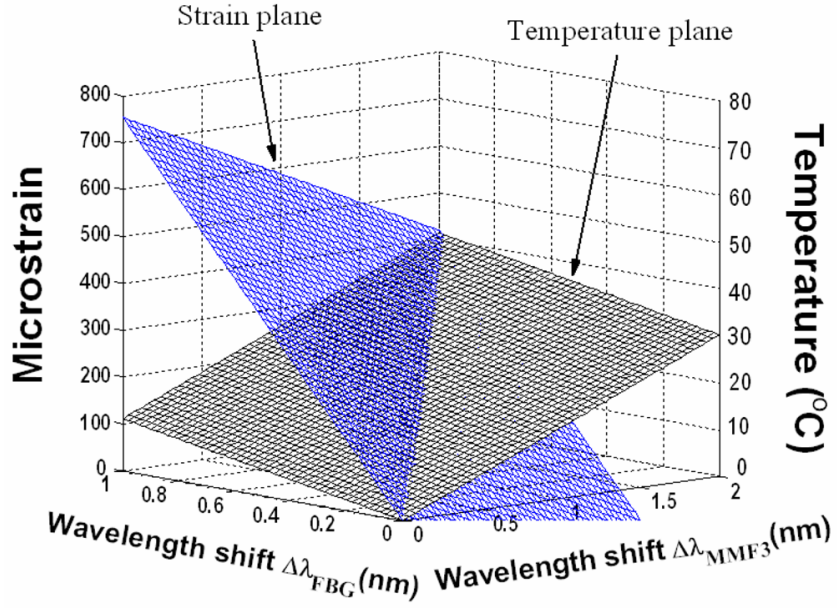

Fig. 5. (Color online) 3D plot illustrating simultaneous measurement of strain and temperature by measuring the wavelength shifts of the FBG and $\mathrm{MMF}_{3}$.

shifts $\Delta \lambda_{\mathrm{FBG}}$ and $\Delta \lambda_{\mathrm{MMF}}$, the strain and temperature can be determined simultaneously.

\section{Conclusion}

A transmission-type simultaneous strain and temperature sensing technique has been demonstrated based on a combination of an FBG and a section of MMF. The applied strain and ambient temperature changes can be obtained by measuring the wavelength shifts of the FBG and the MMF that acts as an MZI. The measurement resolution is very sensitive to the strain and temperature coefficients of the FBG and MMF and their combination. Since the strain and temperature coefficients of MMFs vary with the fiber core sizes and materials, we demonstrated a good combination of $\mathrm{FBG}+\mathrm{MMF}_{3}$ for simultaneous strain and temperature discrimination: $9.21 \mu \varepsilon$ and $0.26^{\circ} \mathrm{C}$ can be achieved for a $10 \mathrm{pm}$ wavelength resolution.

This work is supported by the Natural Science and Engineering Research Council of Canada (NSERC). The authors acknowledge Sumitomo Electric Industries, Ltd. for providing the $\mathrm{MMF}_{3}$.

Table 2. Coefficient Matrices, Matrix Condition Number, and Corresponding Resolutions of Strain and Temperature for the Combination of FBG + MMF $_{i}$

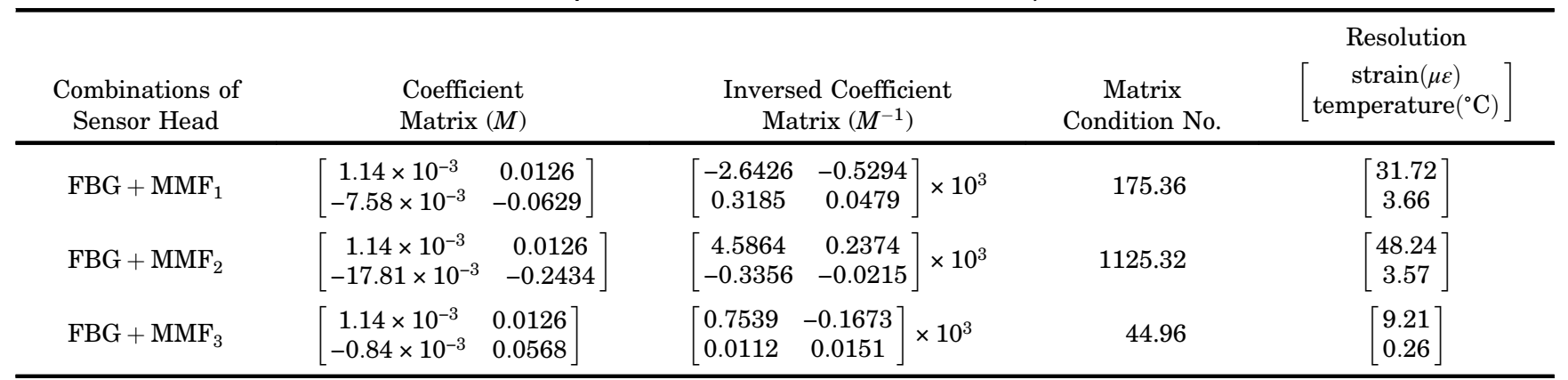




\section{References}

1. F. Farahi, D. J. Webb, J. D. C Jones, and D. A. Jackson, "Simultaneous measurement of temperature and strain: cross-sensitivity considerations,” J. Lightwave Technol. 8, 138-142 (1990).

2. W. Jin, W. C. Michie, G. Thursby, M. Konstantaki, and B. Culshaw, "Simultaneous measurement of strain and temperature: error analysis," Opt. Eng. 36, 598-609 (1997).

3. M. G. Xu, J.-L. Archambault, L. Reekie, and J. P. Dakin, "Simultaneous measurement of strain and temperature using fibre grating sensors," Proc. SPIE 2360, 191-194 (1994).

4. M. G. Xu, J.-L. Archambault, L. Reekie, and J. P. Dakin, "Discrimination between strain and temperature effects using dual-wavelength fibre grating sensors," Electron. Lett. 30, 1085-1087 (1994).

5. E. Chehura, S. W. James, and R. P. Tatam, "Temperature and strain discrimination using a single tilted fibre Bragg grating," Opt. Commun. 275, 344-347 (2007).

6. S. W. James, M. L. Dockney, and R. P. Tatam, "Simultaneous independent temperature and strain measurement using infibre Bragg grating sensors," Electron. Lett. 32, 1133-1134 (1996).

7. P. M. Cavaleiro, F. M. Araújo, L. A. Ferreira, J. L. Santos, and F. Farahi, "Simultaneous measurement of strain and temperature using Bragg gratings written in germanosilicate and boron-codoped germanosilicate fibers," IEEE Photon. Technol. Lett. 11, 1635-1637 (1999).

8. B. O. Guan, H. Y. Tam, H. L. W. Chan, C. L. Choy, and M. S. Demokan, "Discrimination between strain and temperature with a single fiber Bragg grating," Microwave Opt. Technol. Lett. 33, 200-202 (2002).

9. O. Frazão and J. L. Santos, "Simultaneous measurement of strain and temperature using a Bragg grating structure written in germanosilicate fibres," J. Opt. A 6, 553-556 (2004).

10. H. J. Patrick, G. M. Williams, A. D. Kersey, J. R. Pedrazzani, and A. M. Vengsarkar, "Hybrid fiber Bragg grating/long period fiber grating sensor for strain/temperature discrimination," IEEE Photon. Technol. Lett. 8, 1223-1225 (1996).
11. B. O. Guan, H. W. Tam, X. M. Tao, and X. Y. Dong, "Simultaneous strain and temperature measurement using a superstructure fiber Bragg grating," IEEE Photon. Technol. Lett. 12, 675-677 (2000).

12. O. Frazão, R. Romero, G. Rego, P. V. S. Marques, H. M. Salgado, and J. L. Santos, "Sampled fibre Bragg grating sensors for simultaneous strain and temperature measurement," Electron. Lett. 38, 693-695 (2002).

13. O. Frazão, L. M. Marques, and J. M. Baptista, "Fibre Bragg grating interrogation based on high-birefringence fibre loop mirror for strain-temperature discrimination," Microwave Opt. Technol. Lett. 48, 2326-2328 (2006).

14. Q. Wang and G. Farrell, "All-fiber multimode-interferencebased refractometer sensor: proposal and design," Opt. Lett. 31, 317-319 (2006).

15. A. Mehta, W. Mohammed, and E. G. Johnson, "Multimode interference-based fiber-optic displacement sensor," IEEE Photon. Technol. Lett. 15, 1129-1131 (2003).

16. O. Frazão, J. Viegas, P. Caldas, J. L. Santos, F. M. Araújo, L. A. Ferreira, and F. Farahi, "All-fiber Mach-Zehnder curvature sensor based on multimode interference combined with a long-period grating," Opt. Lett. 32, 3074-3076 (2007).

17. Q. Li, C. H. Lin, P. Y. Tseng, and H. P. Lee, "Demonstration of high extinction ratio modal interference in a two-mode fiber and its applications for all-fiber comb filter and hightemperature sensor," Opt. Commun. 250, 280-285 (2005).

18. W. S. Mohammed, P. W. E. Smith, and X. Gu, "All-fiber multimode interference bandpass filter," Opt. Lett. 31, 2547-2549 (2006).

19. E. Li, "Temperature compensation of multimode-interferencebased fiber devices," Opt. Lett. 32, 2064-2066 (2007).

20. Y. Liu and L. Wei, "Low-cost high-sensitivity strain and temperature sensing using graded-index multimode fibers," Appl. Opt. 46, 2516-2519 (2007).

21. P. Sivanesan, J. S. Sirkis, Y. Murata, and S. G. Buckley, "Optimal wavelength pair selection and accuracy analysis of dual fiber grating sensors for simultaneously measuring strain and temperature," Opt. Eng. 41, 2456-2463 (2002). 